\title{
The liver in focus
}

\author{
Michael Roden ${ }^{1,2,3}$
}

Received: 16 December 2015 / Accepted: 18 February 2016 / Published online: 7 April 2016

(C) Springer-Verlag Berlin Heidelberg 2016

Keywords GLP-1 agonists · Hepatokines · Insulin resistance $\cdot$ Lipogenesis $\cdot$ Liver $\cdot$ Mitochondria .

Non-alcoholic fatty liver disease $\cdot$ Thiazolidendiones

\section{Abbreviations \\ NAFLD Non-alcoholic fatty liver disease \\ NASH Non-alcoholic steatohepatitis}

\section{Background}

The liver is not only responsible for handling carbohydrates and lipids, but is also a key signalling organ, producing hormones (hepatokines) and receiving signals from other tissues. Nevertheless, studies on the liver in diabetes mellitus have focused on its role in alterating glucose fluxes (Fig. 1). Although the association of non-alcoholic fatty liver disease (NAFLD) with obesity and type 2 diabetes is very well established, it is only recently that studies have addressed other aspects of hepatic metabolism, particularly lipid fluxes and energy balance. Likewise, the clinical relevance of NAFLD

Michael Roden

michael.roden@ddz.uni-duesseldorf.de

1 Department of Endocrinology and Diabetology, Medical Faculty, Heinrich-Heine University Düsseldorf, c/o Auf'm Hennekamp 65, D-40225 Düsseldorf, Germany

2 Institute for Clinical Diabetology, German Diabetes Center, Leibniz Institute for Diabetes Research at Heinrich Heine University, Düsseldorf, Germany

3 German Center for Diabetes Research (DZD), Düsseldorf, Germany has long been the subject of study. Given its role as a risk factor for hepatocellular carcinoma and cardiovascular diseases, a critical analysis of NAFLD pathophysiology, diagnostic procedures and therapeutic measures is vital to fight this common comorbidity of obesity and type 2 diabetes. The following series of mini-reviews summarises the contributions of leading researchers in this field presented at a symposium at the 51st EASD annual meeting in Stockholm in September 2015.

\section{Pathophysiology}

Despite decades of detailed tracer studies, the complex regulation of metabolic fluxes within the liver has not been fully resolved. In particular, the rapid alterations in pathways controlling endogenous glucose production and lipogenesis during the transition from fasting to feeding and back are not well understood in humans with diabetes and/or NAFLD [1]. As an example, studies in rodent models only recently showed that the effect of insulin to suppress gluconeogenesis and endogenous glucose production is due more to reduced flux of fatty acids resulting from inhibition of adipose tissue lipolysis than to transcriptional regulation of hepatic expression of genes controlling gluconeogenesis [2]. Furthermore, the link between hepatic energy metabolism and abnormal glucose and lipid fluxes has recently been challenged, in that obese insulin resistant humans with NAFLD present with a transient upregulation of their hepatic mitochondrial capacity [3].

As explored by John Jones during this symposium [4], there is a compelling need to develop, validate and apply new magnetic resonance- or stable isotope-based technologies to non-invasively measure fluxes through oxidative, gluconeogenic and lipogenic pathways, and determine their relationship with hepatic energy state in healthy volunteers and diabetes patients. 


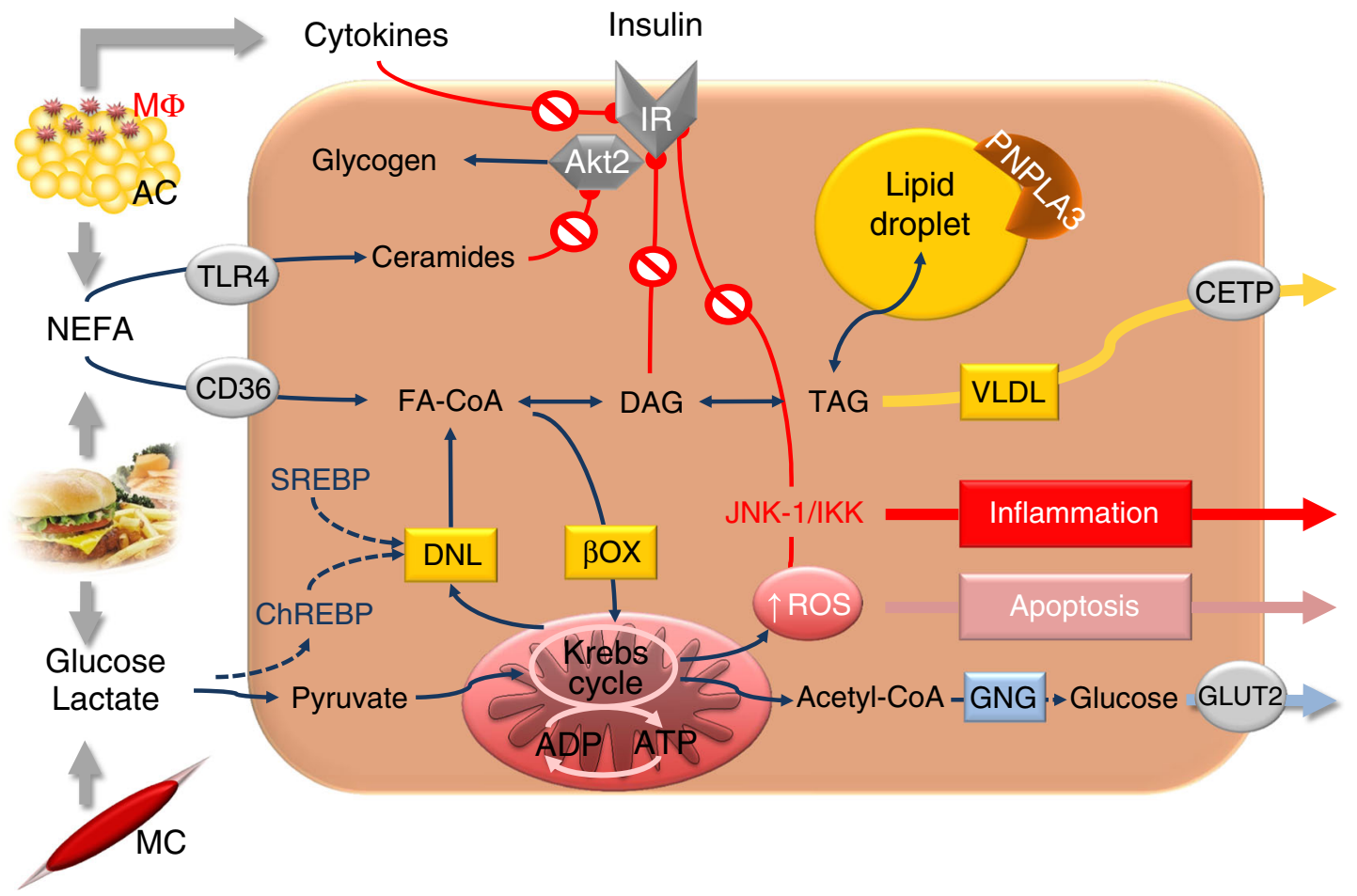

Fig. 1 Cellular mechanisms linking diabetes and metabolic liver diseases. High-energy diets increase the flux of NEFA and glucose to hepatocytes. Likewise, in obesity and insulin resistant states, the flux of NEFA, glucose and lactate is re-directed to the liver, and cytokines mainly originating from inflamed adipose tissue, infiltrated by macrophages $(\mathrm{M} \Phi)$, enter the liver. Within the hepatocytes, fatty acyl-coenzyme A (FA-CoA), via diacylglycerol (DAG) or ceramides, inhibits insulin signalling at the level of the insulin receptor (IR) or Akt2 and thereby causes insulin resistance. Furthermore, the FA-CoA pool, as a precursor of triacylglycerol (TAG), is further enlarged by de novo lipogenesis (DNL), controlled by sterol- and carbohydrate-responsive enhancing binding proteins (SREBP and ChREBP), and decreased by $\beta$ oxidation ( $\beta \mathrm{OX}$ ). TAG

\section{NAFLD and diabetes mellitus}

NAFLD affects $17-46 \%$ of adults and occurs in the majority of obese patients, with at least $10 \%$ of them presenting with non-alcoholic steatohepatitis (NASH) [5]. While a precise diagnosis of steatosis can be made by quantification of liver fat content using ${ }^{1} \mathrm{H}$ magnetic resonance spectroscopy, clinical practice still relies on less exact methods such as ultrasound or measurement of serum transaminases. Even worse, the diagnosis of advanced stages of NAFLD, such as fibrosis and $\mathrm{NASH}$, ultimately requires liver biopsies. Current tools to diagnose NAFLD in the context of diabetes therefore need novel strategies. Several risk scores for NAFLD based on serum biomarkers, transient elastography and genetic analyses may help to improve diagnostic efficacy. In her review in this symposium series, Hannele Yki-Järvinen proposes a new pathogenic classification of NAFLD: the obese/metabolic, the genetic, and the 'double/triple trouble' forms, which would require different screening and follow-up procedures [6]. Indeed, the new EASL-EASD-EASO Clinical Practice can be released in the form of VLDL or stored in lipid droplets, where it undergoes turnover catalysed by hydrolases and acyltransferases such as patatin-like phospholipase domain-containing protein 3 (PNPLA3), which is involved in the development of NAFLD independently of insulin resistance. Enhanced mitochondrial activity can give rise to reactive oxygen species (ROS) and thereby generate oxidative stress with apoptosis and activate inflammatory pathways (c-Jun N-terminal kinase [JNK]/ inhibitor of $\mathrm{kB}$ kinase [IKK]). Finally, accumulation of acetyl-CoA serves as a precursor for gluconeogenesis (GNG) and thereby raises endogenous glucose production and causes hyperglycaemia. AC, adipocyte; CETP, cholesterylester transfer protein; MC, muscle cell; TLR4, Toll-like receptor 4

Guidelines for the management of non-alcoholic fatty liver disease propose a step-wise diagnostic algorithm, which requires joint work-up by diabetologists and hepatologists [5].

\section{Liver-targeted treatments}

Most lifestyle- or drug-based diabetes treatments directly or indirectly affect the liver, for example by targeting an intrahepatic pathway, or by body weight loss reducing liver fat content. However, drugs addressing the selective hepatic insulin resistance of glucose metabolism could favour lipogenesis, and insulin treatment could, by the same mechanism, improve hepatic glucose production without necessarily reducing fat content [7]. By contrast, insulin sensitisers that primarily address adipocyte differentiation, such as thiazolidendiones, lead to marked improvements in NAFLD, but may have side effects limiting their broad use. Treatments primarily addressing NASH rather than diabetes mellitus also have their limitations. A recent Bayesian network meta- 
analysis identified only nine randomised controlled trials of biopsy-confirmed NASH [8]. This study showed moderatequality evidence for the use of thiazolidendiones, pentoxifylline and obeticholic acid to decrease lobular inflammation and for the use of pentoxifylline and obeticholic acid to improve fibrosis. The authors therefore concluded that future comparative trials of combination therapies targeting distinct histological features are warranted.

The review by Kenneth Cusi in this symposium series presents a comprehensive summary of the available treatments for NAFLD in diabetes, but also critically discusses the novel approaches aiming to overcome the limitations of current drugs [9]. In this context, two recent studies provide new hope for the combined treatment of NAFLD, obesity and type 2 diabetes. Liraglutide, which is used to treat both obesity and type 2 diabetes, not only leads to reduction of liver fat content but also to histological resolution of NASH, compared with placebo, suggesting effects in addition to simple weight loss [10]. Finally, preliminary results in rodents suggest that a novel orally available, controlled-release formulation of the protonophore 2,4-dinitrophenol (DNP), an old anti-obesity drug, improves hyperglycaemia, hepatic steatosis and even fibrosis in models of diabetes and NASH [11]. This drug works by inducing mild mitochondrial uncoupling, and further investigations in this area may help explain the recently reported dynamic abnormalities of hepatic mitochondrial functionality in humans with NAFLD [3].

\section{Outlook and open questions}

The rapid progress being made on the interaction between the liver and diabetes will hopefully lead to solutions to key questions such as: (1) 'which are the primary metabolic abnormalities underlying this interaction?', (2) 'what are the most efficient procedures for screening and diagnosis?', and (3) 'which novel targets for resolving hepatic insulin resistance and NAFLD can be safely translated into clinical practice?'

Funding During the last 7 years, the work of MR has been supported by the Ministry of Science and Research of the State of North RhineWestphalia (MIWF NRW) and the German Federal Ministry of Health (BMG) as well as by a grant from the Federal Ministry for Research (BMBF) to the German Center for Diabetes Research (DZD e.V.), and by grants from the European Union, the European Foundation for the
Study of Diabetes (EFSD), the Helmholtz Alliance to Universities (ICEMED), the German Research Foundation (DFG), the German Diabetes Association (DDG) and the Schmutzler-Stiftung.

Duality of interest MR declares that there is no duality of interest associated with this manuscript.

Contribution statement MR is responsible for the conception of this manuscript, drafting the article and approving the final version for publication.

\section{References}

1. Koliaki C, Roden M (2013) Hepatic energy metabolism in human diabetes mellitus, obesity and non-alcoholic fatty liver disease. Mol Cell Endocrinol 379:35-42

2. Titchenell PM, Chu Q, Monks BR, Birnbaum MJ (2015) Hepatic insulin signalling is dispensable for suppression of glucose output by insulin in vivo. Nat Commun 6:7078

3. Koliaki C, Szendroedi J, Jelenik T et al (2015) Adaptation of hepatic mitochondrial function in humans with non-alcoholic fatty liver or steatohepatitis. Cell Metab 21:739-746

4. Jones JG (2016) Hepatic glucose and lipid metabolism. Diabetologia doi:10.1007/s00125-016-3940-5

5. European Association for the Study of the Liver (EASL), European Association for the Study of Diabetes (EASD) and European Association for the Study of Obesity (EASO) (2016) EASLEASD-EASO Clinical Practice Guidelines for the management of non-alcoholic fatty liver disease. Diabetologia doi:10.1007/s00125016-3902-y

6. Yki-Järvinen H (2016) Diagnosis of non-alcoholic fatty liver disease (NAFLD). Diabetologia doi:10.1007/s00125-016-3944-1

7. Anderwald C, Bernroider E, Krssak M et al (2002) Effects of insulin treatment in type 2 diabetic patients on intracellular lipid content in liver and skeletal muscle. Diabetes 51:3025-3032

8. Singh S, Khera R, Allen AM, Murad MH, Loomba R (2015) Comparative effectiveness of pharmacological interventions for nonalcoholic steatohepatitis: a systematic review and network meta-analysis. Hepatology 62:1417-1432

9. Cusi K (2016) Treatment of patients with type 2 diabetes and nonalcoholic fatty liver disease: current approaches and future directions. Diabetologia doi:10.1007/s00125-016-3952-1

10. Armstrong MA, Gaunt P, Aithal GP et al (2016) Liraglutide safety and efficacy in patients with non-alcoholic steatohepatitis (LEAN): a multicentre, double-blind, randomised, placebo-controlled phase 2 study. Lancet 387:679-690

11. Perry RJ, Zhang D, Zhang XM, Boyer JL, Shulman GI (2015) Controlled-release mitochondrial protonophore reverses diabetes and steatohepatitis in rats. Science 347:1253-1256 